\title{
Pressure-sensor-based Gait Analysis for Disabled People
}

\author{
Yeou-Jiunn Chen, ${ }^{1}$ Chung-Min Wu, ${ }^{2}$ Pei-Chung Chen, ${ }^{3}$ \\ Aaron Raymond See, ${ }^{1}$ and Shih-Chung Chen ${ }^{1 *}$ \\ ${ }^{1}$ Department of Electrical Engineering, Southern Taiwan University of Science and Technology, \\ No. 1, Nan-Tai Street, Yungkang Dist., Tainan City 710301, Taiwan \\ ${ }^{2}$ Department of Intelligent Robotics Engineering, Kun Shan University, \\ No. 195, Kunda Rd., Yongkang Dist., Tainan City 710303, Taiwan \\ ${ }^{3}$ Department of Mechanical Engineering, Southern Taiwan University of Science and Technology, \\ No. 1, Nan-Tai Street, Yungkang Dist., Tainan City 710301, Taiwan
}

(Received May 29, 2021; accepted December 2, 2021)

Keywords: pressure sensor, gait disorder, gait analysis

The issue of aging is a complex social challenge. When elderly people suffer from diseases such as hemiplegia, their quality of life is greatly affected. The only way to reduce the impact of such diseases is to perform long-term rehabilitation, which is extremely time-consuming. Thus, gait information for clinical practice can effectively help subjects with stroke and their occupational therapists. In this study, a pressure-sensor-based gait analysis system is proposed to help occupational therapists evaluate the gait of disabled people. To effectively acquire gait information without affecting the user's activities, the insole of the foot is selected as the pressure-sensing interface. To accurately acquire information of the plantar pressure, forcesensing resistor (FSR) film pressure sensors (FPSs) are used. To greatly reduce the differences between the outputs of FSR FPSs, linear regression is used to normalize the outputs. To correctly detect the steps in the gait cycle, an endpoint detection algorithm is applied. The experimental results showed that the proposed normalization process can greatly reduce the differences between the outputs of FSR FPSs. Moreover, occupational therapists agreed that the proposed system can help them evaluate the gait of users.

\section{Introduction}

The issue of aging is a complex social challenge of great concern. ${ }^{(1,2)}$ Diseases such as hemiplegia have a high incidence and recurrence rate among elderly people. These diseases are generally caused by a brain lesion, such as a tumor, or by stroke, causing paralysis to one side of the body and reducing the quality of life of elderly people. To reduce the impact of these diseases, long-term rehabilitation must be performed, which is extremely time-consuming. Occupational therapists require gait information to evaluate the effectiveness of rehabilitation and adjust the training activities accordingly. Thus, gait information for clinical practice is very useful for subjects with stroke and their occupational therapists.

*Corresponding author: e-mail: chung@stust.edu.tw

https://doi.org/10.18494/SAM3559 
When elderly people suffer from non-fatal brain diseases (such as tumors) or strokes, about $70 \%$ of them become disabled, of which approximately $40 \%$ will become severely disabled. ${ }^{(3)}$ The effects of these diseases on subjects are often reflected in their gait. Therefore, it is important to preserve the quality of life of subjects by restoring their walking ability. According to a recent study, the main causes of gait abnormalities are sensory impairment (18\%), spinal cord disease (17\%), repetitive stroke (15\%), unknown causes (14\%), and Parkinson's disease $(12 \%){ }^{(4)}$ To help subjects with such diseases, occupational therapists need to accurately assess their gaits then provide professional advice about activities to improve their gait. Thus, a gait analysis system could effectively reduce the effort of occupational therapists and increase the effectiveness of rehabilitation for subjects.

Over the last decade, many devices that can be used to detect motion have been developed, including Northern Digital OPTOTRAK, Isolatek WB-4, Microsoft Kinect, Vicon NDI, Polhemus Liberty 240/8, Hexamite HX17, and Organic Motion STAGE. ${ }^{(5-12)}$ However, the user's environment is usually complex and includes many interference factors that could cause many motion detection methods to fail, such as furniture, space layout, people moving, and sound and light sources. Therefore, a robust motion detection method could greatly reduce the effects of interference factors and increase the value of gait analysis systems.

Many studies have used inertial sensing technology, which is an innovative motion tracking system, to improve the convenience of gait analysis systems. ${ }^{(12-17)}$ Inertial sensors are used to measure the user's gait parameters, such as joint tilt angle, walking speed, step length, stride length, and relative swing position. However, inertial sensing technology requires many sensors to be installed on the user, which is uncomfortable for the user and hinders their actions. Thus, a simplified and convenient gait analysis system could greatly increase the user's acceptance of such technology. The ability to acquire gait information without affecting the user's activities could help occupational therapists to correctly evaluate the gait of subjects and provide effective training activities.

Many researchers have developed plantar-pressure-sensing interfaces to correctly obtain the gait information of users without affecting their activities. ${ }^{(18-24)}$ The insole of the foot has successfully been used as an interface between the ground and the human body, and the information derived from foot plantar pressure measurement is important in evaluating gait. F-Scan and Kistler can obtain gait information, including the peak plantar pressure, the position of the central pressure, and the ground reaction force. ${ }^{(18,19)}$ However, the equipment is extremely expensive and cannot be used at home for a long time. Some researchers have developed plantar pressure insoles with flexible pressure sensors. ${ }^{(20,21)}$ Using a high number of pressure sensors, the changes in pressure during walking can be effectively detected. However, the price of such products is very high. To reduce the cost, Hamid et al. and Fei et al. used fewer pressure sensors to obtain gait information. ${ }^{(22,23)}$ Nevertheless, they only evaluated standing, squatting, and forward and backward movements, and did not analyze the supporting strength of the feet. Thus, their systems cannot be adopted by occupational therapists. Therefore, an inexpensive plantarpressure-sensing interface that can measure the supporting strength of a single limb could effectively improve the effectiveness of rehabilitation.

In this study, a pressure-sensor-based gait analysis system is proposed to help occupational therapists evaluate the gait of disabled people. To effectively acquire gait information without 
affecting the user's activities, the insole of the foot is selected as the pressure-sensing interface. To effectively acquire the plantar pressure, force-sensing resistor (FSR) film pressure sensors (FPSs) are used. To greatly reduce the differences between the outputs of FSR FPSs, a secondorder linear function estimated using the least squares method is applied to normalize the outputs of different FSR FPSs. To correctly detect the steps in the gait cycle, an endpoint detection algorithm is developed.

\section{Pressure-sensor-based Gait Analysis System}

The proposed pressure-sensor-based gait analysis system is described in detail as follows.

\subsection{Design of insole of the feet}

In the clinical practice of occupational therapists, there are three main aspects in the evaluation of the walking ability of subjects with gait disorder:(25)

- ataxic gait: whether the patient has freezing of gait;

- limb stability: the characteristics of swinging gait, dragging legs, low walking speed, short stride, stiffness, reduced step height, hesitation in walking, etc.;

- the symmetry of gait: the symmetry of walking with both feet and the range of variability.

To obtain clinical gait information, the gait cycle is analyzed as shown in Fig. 1. From the results of the gait cycle analysis and the clinical practice of occupational therapists, the landing time and supporting strength of the left foot, right foot, and both feet during walking should be obtained. In addition, the difference in supporting strength between the two feet and the change in the supporting strength of a single foot are important in the evaluation of gait. Therefore, the pressure-sensing insole shown in Fig. 2 is designed.

Using the pair sensors (R1, R5) and (L1, L5), the corresponding supporting time and strength of one foot can be easily detected. Using the pair sensors (R2, R3) and (L2, L3), the change in the supporting strength of one foot can be detected and used to estimate the step balance of a subject. Ten sensors can meet the requirements for the clinical evaluation of gait by occupational therapists. The use of such pressure-sensing insoles can also greatly reduce the cost of gait analysis systems.

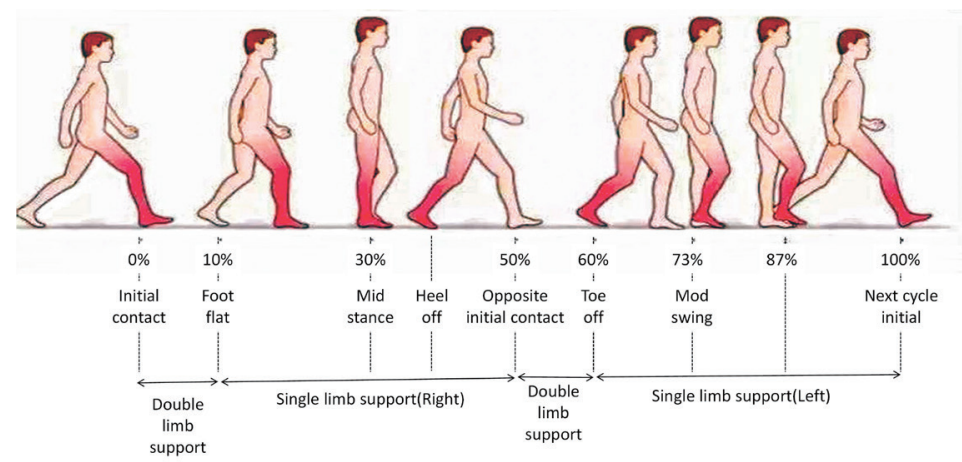

Fig. 1. (Color online) Analysis of gait cycle. 


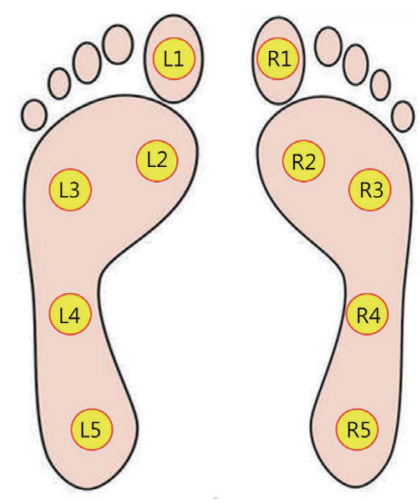

Fig. 2. (Color online) Positions of sensors on the insoles.

\subsection{Hardware design}

The architecture of the proposed pressure-sensor-based gait analysis system is shown in Fig. 3. First, the sensors used on the plantar pressure insole should provide repeatable and stable performance, be easy to integrate, and not hinder the user's activities. Moreover, the insole should be comfortable to improve user acceptance. Compared with other types of pressure sensors, FSR FPSs have light weight, high sensitivity, fast dynamic response, and good repeatability. Therefore, FSR FPSs are used in the plantar pressure insole to meet the requirements of clinical gait analysis.

When a subject is walking, the pressure on the insole changes, which changes the resistance of the FSR FPS. Therefore, a resistance-voltage signal conversion circuit (as shown in Fig. 4) is designed to detect the voltage signal, which represents the pressure on the insole, from the insole sensors. The output voltage $\left(V_{\text {out }}\right)$ associated with the pressure can be defined as

$$
V_{\text {out }}=\frac{V_{c c} R}{R_{F S R}+R},
$$

where $V_{c c}$ is the input voltage of the conversion circuit. $R_{F S R}$ and $R$ are the resistances of the FSR FPS and reference sensor, respectively. Generally, the resistances for different resistors are considerably different and it is very difficult to normalize them using hardware. Therefore, a normalization process is implemented using a microcontroller as described in detail in the next subsection.

\subsection{Gait signal processing}

To reduce the error caused by using different resistors, a normalization process is implemented using a second-order linear function. Each sensor in the plantar pressure insole is tested using standard weights; a weight test is performed by adding weights in units of $1 \mathrm{~kg}$ from 0 to $10 \mathrm{~kg}$ to the FSR FPSs in the plantar pressure insole to obtain the corresponding outputs of the sensors. To normalize the difference between the outputs of the FSR FPSs, an FSR FPS is 


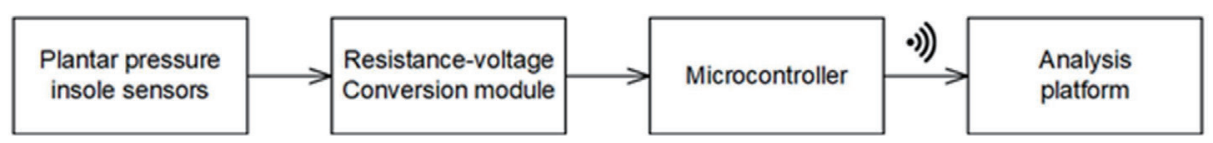

Fig. 3. Architecture of the pressure-sensor-based gait analysis system.
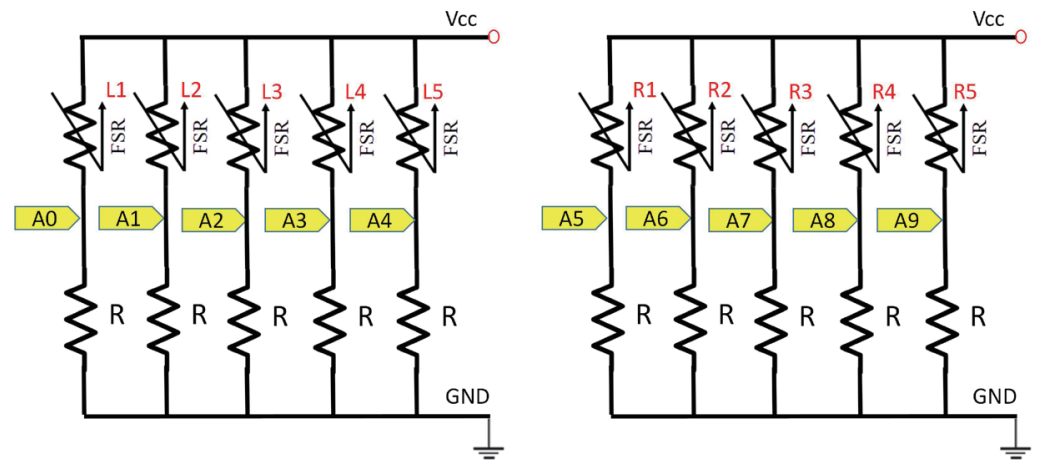

Fig. 4. (Color online) Resistance-Voltage signal conversion circuit.

selected as a reference sensor. The relations between the outputs of the other FSR FPSs $x$ and that of the reference sensor are modeled as second-order linear functions $f$ defined as

$$
f(x)=\beta_{0}+\beta_{1} x+\beta_{2} x^{2},
$$

where $\beta_{0}, \beta_{1}$, and $\beta_{2}$ are the coefficients of the second-order linear function and are estimated by the least squares method. Using this function, the outputs of the FSR FPSs can be normalized to the same output range. After the outputs of the FSR FPSs are normalized, a second-order linear function is estimated by the least squares method and used to transform the voltage to a mass in kilograms, which occupational therapists are familiar with.

After the gait signal has been normalized and transformed, the following endpoint detection algorithm is applied to find the steps in the gait cycle:

Step 1: Compute the average magnitude of the gait signal as well as the average magnitude of the noise. Before the foot plantar pressure is used, the average magnitude of the noise is obtained from the first $100 \mathrm{~ms}$ of the gait signal. The mean and standard deviation of the average magnitude of noise are calculated and used to determine their thresholds to separate the actual gait signal from the noise.

Step 2: At the beginning of the signal, the first point where the signal magnitude exceeds the previously set threshold for the average magnitude is searched for. This location marks the beginning of the gait signal.

Step 3: From this point, a forward search is carried out until the magnitude drops below a lower magnitude threshold. This location marks the end of the gait signal.

Step 4: The above process is repeated, starting from the end of the gait signal, to locate the beginning and end of the next gait signal. 


\section{Experimental Results}

In this study, eight normal subjects and two disabled subjects who had experienced stroke were asked to participate in experiments to evaluate the performance of our proposed system. The experimental results are detailed in the following.

\subsection{Results of sensor normalization}

As described in this subsection, the FSR FPSs are normalized. The results before normalization are shown in Fig. 5(a). In this figure, it is clear that the outputs of the FSR FPSs are different at the same pressure, especially for heavy inputs. To reduce the difference between the outputs of the FSR FPSs, the second-order linear functions are used to normalize the outputs, and the results are shown in Fig. 5(b). It is clear that the proposed approach can effectively reduce the difference between the outputs of the FSR FPSs and ensure their consistency.

To evaluate the performance of the proposed normalization process, we evaluated the ranges of the outputs of FSR FPSs at different pressures $(\mathrm{kg})$, as shown in Fig. 6. Before the normalization, the range of outputs, defined as the difference between the maximum and minimum voltages, was between 45 and $85 \mathrm{mV}$. After normalization, the range was greatly reduced to between 11 and $31 \mathrm{mV}$, thus reducing the error by between 50.9 and $77.8 \%$. Thus, the

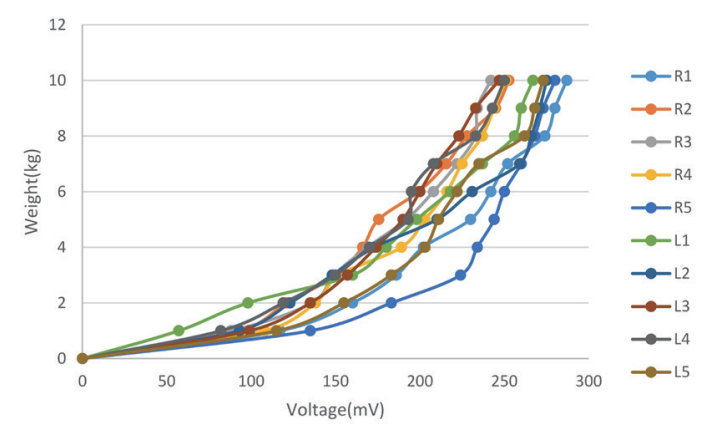

(a)

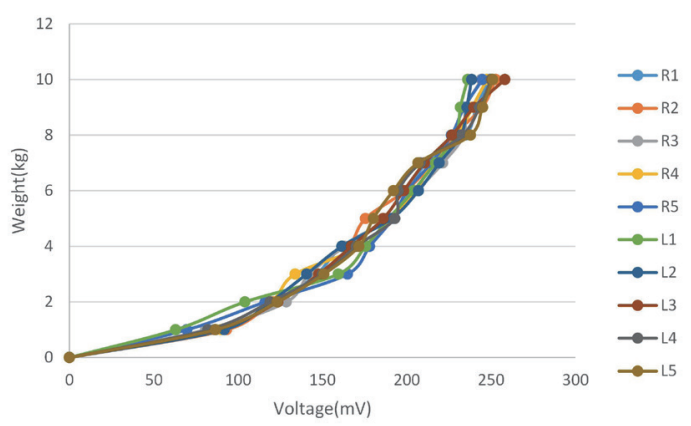

(b)

Fig. 5. (Color online) Transform functions (a) before and (b) after normalization.

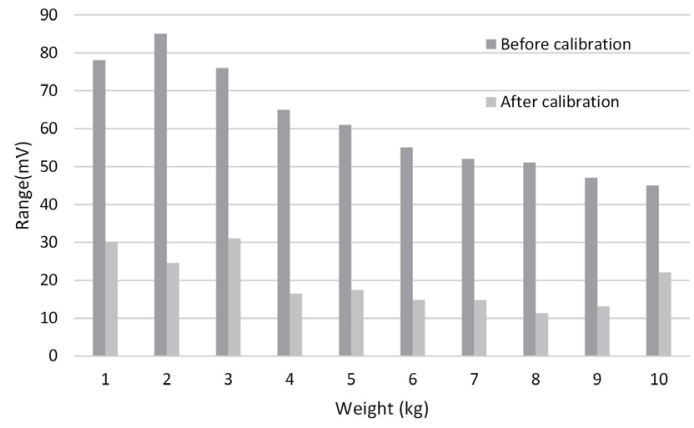

Fig. 6. Ranges for the outputs of FSR FPSs at different pressures. 
proposed normalization process can successfully improve the accuracy of plantar pressure insoles.

\subsection{Results of gait information analysis}

In this subsection, the gait information acquired by the proposed gait analysis system is examined. A normal subject and a disabled subject were examined and the results are shown in Figs. 7 and 8, respectively. In Fig. 7, the steps can be easily detected. Moreover, the fifth sensor

(a)

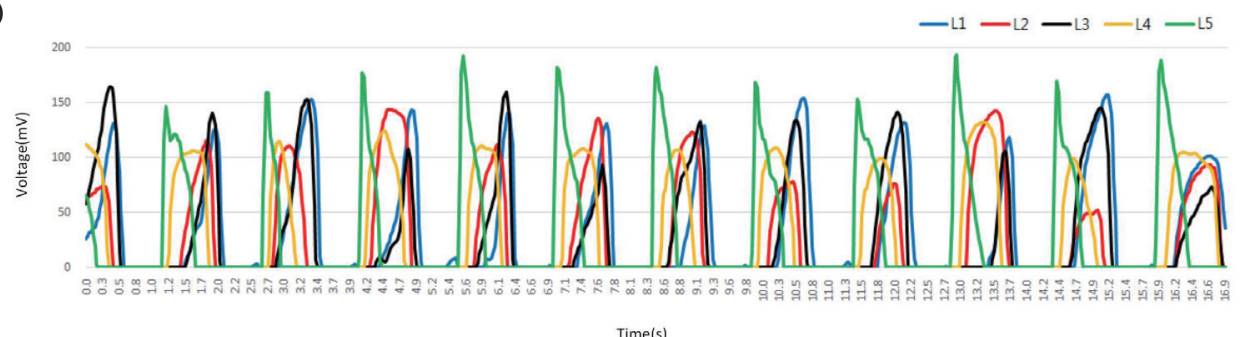

(b)

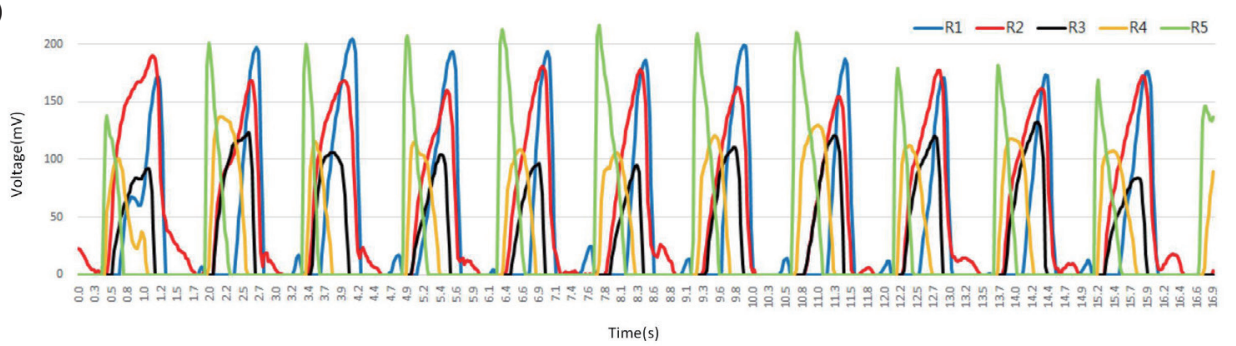

Fig. 7. (Color online) Gait information of (a) left and (b) right feet of normal subject.

(a)

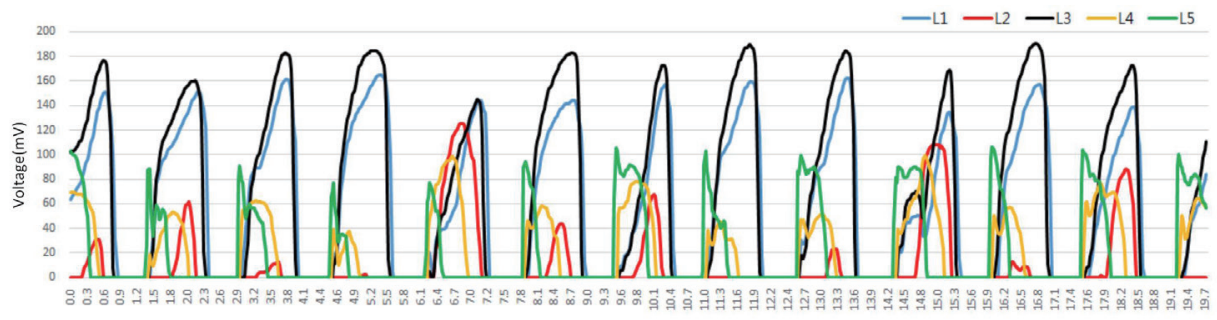

Time(s)

(b)

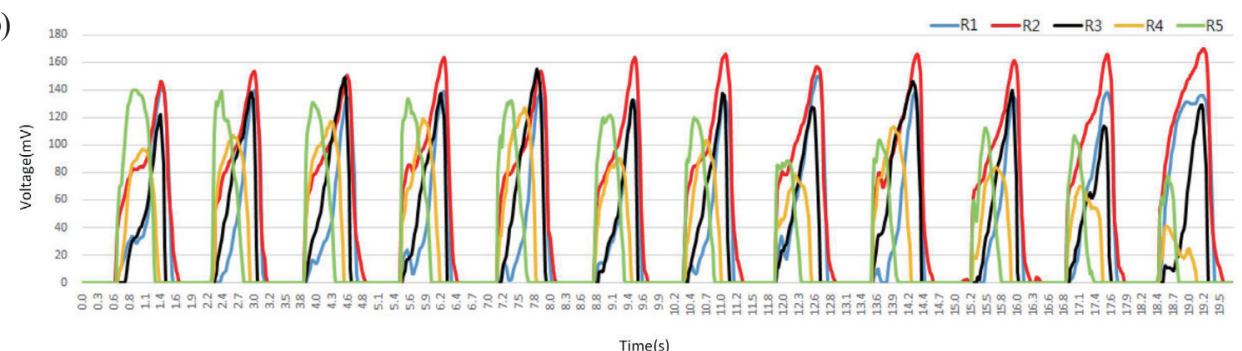

Fig. 8. (Color online) Gait information of (a) left and (b) right feet of disabled subject. 
of the left and right feet is activated first and the last activated sensor is the first sensor of the left and right feet. This behavior meets the characteristic of steps in the gait cycle. In Fig. 8, the gait information of the disabled subject, who had experienced a mild stroke, is considerably different from that in Fig. 7. The left foot of the disabled subject cannot work well and the supporting strength of the left foot is relatively uneven. This is due to the excessive stiffness of the hip/knee joints, which causes insufficient forward thrust of the thigh or the foot plate. Therefore, the disabled subject generates an uneven force after the foot falls.

To objectively evaluate the proposed approach, all 10 subjects were asked to walk a distance of $10 \mathrm{~m}$. A total of 201 testing data were collected. The supporting gait cycle was manually labeled to evaluate the performance of the proposed endpoint detection algorithm. The recognition rates for normal subjects and subjects with stroke are 95.47 and $91.56 \%$, respectively. In this experiment, an error always occurs when the FSR FPSs are slightly pressed by the pressure of the shoes. Moreover, the accuracy for two disabled subjects is slightly lower, mainly because the foot sometimes drags slightly when a disabled subject is walking.

Finally, the gait information required by occupational therapists was examined by using a normal subject and a disabled subject. For clinical practice, occupational therapists need the supporting strength and the ratio of the stance phase to the swing phase. For the normal subject, the durations of the gait cycle for the left and right feet are 1.35 and $1.31 \mathrm{~s}$, respectively. For the disabled subject, the durations of the gait cycle for the left and right feet are both 1.65 s, i.e., the gait cycle durations of the left and right feet are almost the same. The proposed system shows that the walking speed of the disabled subject is lower than that of the normal subject.

However, for the normal subject, the durations of the stance phase for the left and right feet are 56.85 and $58.02 \%$, compared with 66.75 and $52.36 \%$ for the disabled subject, respectively. It is clear that the duration of the stance phase for the subject with stroke is different for the left and right feet. The subject with stroke examined in this study always walks more heavily on the left foot. We demonstrated our system to occupational therapists, who considered that the gait information obtained for the system will be useful for clinical practice.

\section{Conclusion}

In this study, a pressure-sensor-based gait analysis system was successfully developed to help occupational therapists evaluate the gait of disabled people. The insole of the feet can be used as a pressure-sensing interface to effectively acquire information of the plantar pressure without affecting the user's activities. FSR FPSs can be used to effectively acquire the plantar pressure. By using linear regression to normalize the outputs of FSR FPSs, the difference between the outputs can be greatly reduced. The endpoint detection algorithm is able to correctly detect the steps in the gait cycle. Our experimental results showed that the proposed normalization process greatly reduced the difference between the outputs of FSR FPSs. Moreover, occupational therapists agreed that the proposed system can help them evaluate the gait of users. In the future, the proposed approach should be examined for a larger number of subjects. 


\section{Acknowledgments}

This work was supported by the Ministry of Science and Technology (MOST), Taiwan, under Grants MOST 108-2221-E-168-008-MY2, MOST 108-2221-E-218-017-MY2-02, and MOST 108-2221-E-218-018-MY2. We are indebted to all study participants and members of the research team.

\section{References}

1 J. Feng, G. Hong, W. Qian, R. Hu, and G. Shi: Sustainability 12 (2020) 5086. https://doi.org/10.3390/su12125086

2 Y. Han, Y. He, J. Lyu, C. Yu, M. Bian, and L. Lee: Global Health J. 4 (2020) 11. https://doi.org/10.1016/j. glohj.2020.01.002

3 J. S. Rong: Practical Hemiplegia Rehabilitation Technical Illustration (People's Medical Publishing House, USA, 2005).

4 A. Aboutorabi, M. Arazpour, M. Bahramizadeh, S. W. Hutchins, and R. Fadayevatan: Aging Clin. Exp. Res. 28 (2016) 393. https://doi.org/10.1007/s40520-015-0420-6

5 J. Zhou and Y. Q. Yu: Ind. Rob. 38 (2011) 172. https://doi.org/10.1108/01439911111106381

6 S. Cosentino, K. Petersen, Z. Lin, L. Bartolomeo, S. Sessa, M. Zecca, and A. Takanishi: Adv. Rob. 28 (2014) 781. https://doi.org/10.1080/01691864.2014.889577

7 A. Pfister, A. M. West, S. Bronner, and J. A. Noah: J. Med. Eng. Technol. 38 (2014) 274. https://doi.org/10.3109 /03091902.2014.909540

8 L. D. Duffell, N. Hope, and A. H. McGregor: Proc. Inst. Mech. Eng. Part H 228 (2014) 206. https://doi. org $/ 10.1177 / 0954411913518747$

9 Aurora Electromagnetic Measurement System: https://www.ndigital.com/products/aurora/ (accessed July 2021).

10 Polhemus Inc.: http://www.polhemus.com (accessed July 2021).

11 Ultrasonic Industrial Positioning Systems: http://www.hexamite.com (accessed July 2021).

12 X. Robert-Lachaine, H. Mecheri, C. Larue, and A. Plamondon: Gait Posture 54 (2017) 80. https://doi. org/10.1016/j.gaitpost.2017.02.029

13 X. Robert-Lachaine, H. Mecheri, C. Larue, and A. Plamondon: Med. Biol. Eng. Comput. 55 (2017) 1. https:// doi.org/10.1007/s11517-016-1537-2

14 T. Sun, Q. Liu, W. Li, Z. Lu, H. Chen, P. Chen, Z. Lu, C. Wei, A. Hou, C. Wang, M. Li, Q. Liu, L. Duan, Z. Wu, and Y. Shen: Proc. 2016 IEEE Int. Conf. Information and Automation (ICIA 2016) 1612-1617. https://doi. org/10.1109/ICInfA.2016.7832076.

15 Y. Gao, Z. Jiang, W. Ni, Z. L. Vasic, M. Cifrek, M. Du, M. I. Vai, and S. H. Pun: Proc. 2017 Int. Conf. Medical and Biological Engineering (CMBEBIH 2017) 300. https://doi.org/10.1007/978-981-10-4166-2 45.

16 C. Tunca, N. Pehlivan, N. Ak, B. Arnrich, G. Salur, and C. Ersoy: Sensors 17 (2017) 825. https://doi.org/10.3390/ s17040825

17 C. Nüesch, E. Roos, G. Pagenstert, and A. Mündermann: J. Biomech. 57 (2017) 32. https://doi.org/10.1016/j. jbiomech.2017.03.015.

18 P. Cavanagh and J. Ulbrecht: Foot 4 (1994) 123. https://doi.org/10.1016/0958-2592(94)90017-5.

19 A. N. Leunkeu, T. Lelard, R. J. Shephard, P. L. Doutrellot, and S. Ahmaidi: NeuroRehabilitation 35 (2014) 657. https://doi.org/10.3233/NRE-141163

20 Pedar Novel System: http://www.novel.de/products/pedar (accessed July 2021).

21 Tekscan Gait Analysis System: http://www.tekscan.com (accessed July 2021).

22 R. Hamid, S. Wijesundara, L. McMillan, D. Scott, J. M. Redoute, P. R. Ebeling, and M. R. Yuce: Proc. 2017 Annu. Int. Conf. IEEE Engineering in Medicine and Biology Society (IEEE EMBS 2017) 139. https://doi. org/10.1109/EMBC.2017.8036781.

23 F. Fei, Y. Leng, M. Yang, C. Wu, and D. Yang: Proc. 2019 IEEE 2nd Int. Conf. Micro/Nano Sensors for AI, Healthcare, and Robotics (NSENS 2019) 506. https://doi.org/10.1109/NSENS49395.2019.9293994.

24 S. Crea, M. Donati, S. M. M. De Rossi, C. M. Oddo, and N. Vitiello: Sensors 14 (2014) 1073. https://doi. org/10.3390/s140101073.

25 E. Dolatabadi, A. Mansfield, K. K. Patterson, B. Taati, and A. Mihailidis: IEEE J. Biomed. Health Inf. 21 (2017) 1297. https://doi.org/10.1109/JBHI.2016.2633000. 


\section{About the Authors}

Yeou-Jiunn Chen received his B.S. degree in mathematics from Tatung Institute of Technology, Taipei, Taiwan, and his Ph.D. degree from the Institute of Information Engineering, National Cheng Kung University, Tainan, Taiwan, in 1995 and 2000, respectively. He was with the Advanced Technology Center, Computer and Communications Laboratories, Industrial Technology Research Institute, from 2001 to 2005 as a researcher. He is currently a professor at the Department of Electrical Engineering, Southern Taiwan University of Science and Technology, Tainan, Taiwan. His research interests include biomedical signal processing, spoken language processing, and artificial intelligence. Dr. Chen is a member of the Biomedical Engineering Society, Taiwan Rehabilitation Engineering and Assistive Technology Society, and the Association for Computational Linguistics and Chinese Language Processing. (chenyj@stust.edu.tw)

Chung-Min Wu received his B.S. degree in automatic control engineering from Feng Chia University, Taichung, Taiwan, his M.S. degree in biomedical engineering from National Cheng Kung University, Tainan, Taiwan, and his Ph.D. degree in electrical engineering from National Cheng Kung University in 1994, 1998, and 2004, respectively. He is an associate professor in the Department of Intelligent Robotics Engineering, Kun Shan University, Taiwan. His research interests include fuzzy control, biomedical signal processing, and human-computer interaction. $\mathrm{He}$ is a member of the Taiwanese Society of Biomedical Engineering and Taiwan Rehabilitation Engineering and Assistive Technology Society. (cmwu@mail.ksu.edu.tw)

Pei-Chung Chen received his M.S. and Ph.D. degrees in mechanical engineering from National Cheng Kung University, Taiwan, in 1988 and 1993, respectively. He is an associate professor in the Department of Mechanical Engineering, Southern Taiwan University of Science and Technology. In 2006, he joined a group specializing in medical assistive devices and started research on mobility assistive devices. He is the director of the Medical Mobility Assist Center. His research interests include smart mobility assistive devices, neural fuzzy control, intelligent control, and gasoline engine fault diagnosis. He is a member of the Taiwanese Society of Biomedical Engineering and Taiwan Rehabilitation Engineering and Assistive Technology Society. (chenpc@stust.edu.tw)

Aaron Raymond See is an assistant professor in the Department of Electrical Engineering, Southern Taiwan University of Science and Technology. He concurrently holds the position of Director of Student Affairs in the Office of International Affairs. He was born in Manila, Philippines, and received his B.S. degree in electronics and communications engineering from De La Salle University, Manila, in 2006. He obtained his master's and Ph.D. degrees in electrical engineering with a major in biomedical engineering at Southern Taiwan University of Science in Technology in 2010 and 2014, respectively. He handles courses in assistive technology device design and engineering education curriculum transformation. His research interests are in assistive device design and development, biomedical image and signal processing, and engineering education. (aaronsee@stust.edu.tw) 
Shih-Chung Chen received his B.S. degree from the Department of Electrical Engineering, Feng Chia University, Taichung, Taiwan, his M.S. degree from the Institute of Control Engineering, National Chiao Tung University, Hsinchu, Taiwan, and his Ph.D. degree in electrical engineering from National Cheng Kung University, Tainan, Taiwan, in 1982, 1988, and 2000, respectively. He is a professor and has been with the Department of Electrical Engineering, Southern Taiwan University of Science and Technology. His research interests include braincomputer interfaces, biomedical signal processing, system integration, and assistive device implementation. He is a member of the Taiwanese Society of Biomedical Engineering and Taiwan Rehabilitation Engineering and Assistive Technology Society. (chung@stust.edu.tw) 